\title{
GT2005-68365
}

\section{NUMERICAL HEAT TRANSFER ANALYSIS OF AN INNOVATIVE GAS TURBINE COMBUSTOR: COUPLED STUDY OF RADIATION AND COOLING IN THE UPPER PART OF THE LINER}

\author{
A. Andreini, A. Bacci, C. Carcasci, B. Facchini \\ Dipartimento di Energetica "Sergio Stecco" \\ Università degli Studi di Firenze \\ Street Santa Marta, 3 \\ 50139 Firenze - ITALY \\ Email: bacci@brun.de.unifi.it
}

\author{
A. Asti, G. Ceccherini, E. Del Puglia, R. Modi \\ GE Energy Oil \& Gas, Nuovo Pignone \\ Street F. Matteucci, 2, 50100, Firenze, Italy \\ Email: Antonio.Asti@ge.com
}

\begin{abstract}
A numerical study of a single can combustor for the GE10 heavy-duty gas turbine, which is being developed at GE-Energy (Oil \& Gas), is performed using the STAR-CD CFD package. The topic of the present study is the analysis of the cooling system of the combustor liner's upper part, named "cap".

The study was developed in three steps, using two different computational models. As first model, the flow field and the temperature distribution inside the chamber were determined by meshing the inner part of the liner. As second model, the impingement cooling system of the cold side of the cap was meshed to evaluate heat transfer distribution. For the reactive calculations, a closure of the BML (Bray-Moss-Libby) approach based on Kolmogorov-Petrovskii-Piskunov theorem was used. The model was implemented in the STAR-CD code using its user coding features. Then the radiative thermal load on the liner walls was evaluated by means of the STAR-CD-native Discrete Transfer model.

The selection of the radiative properties of the flame was performed using a correlation procedure involving the total emissivity of the gas, the mean beam length and the gas temperature.

The estimated heat flux on the cap was finally used as boundary condition for the calculation of the cooling system, consisting of 68 staggered impingement jet lines on the cold side of the cap.

The resulting temperature distribution shows a good agreement with the experimental values measured by thermocouples. The results confirm the validity of the implemented procedure,
\end{abstract}

and point out the importance of a full CFD computation as an additional tool to support classic correlation design procedures.

\section{INTRODUCTION}

In the design of a modern low- $N O_{x}$ combustor for gas turbines, many different aspects must be taken into account at the same time. The need of low emissions leads to the development of new pilot systems capable of stabilizing leaner and leaner main flames. In parallel, the air management has to become more precise.

In the old combustor of the GE10 machine, the main flame is stabilized by a small amount of fuel injected at the throat of the cap (figure 1). Since this fuel burns in a diffusive way, its $N O_{x}$ production is relatively high and heavily impacts the overall $N O_{x}$ emissions of the combustor. One useful idea to overcome this problem is to replace the existing pilot system with a new one of a different nature: partially premixed.

Such idea has been implemented in the new combustor examined in this paper. The pilot fuel is injected in a series of premixed microburners located at the throat of the cap (see figure 2 for the location, see paragraph 1.2 for specific details) so that the resulting flames have a lower peak temperature but similar capabilities of stabilizing the main flame. To make the combustion of these premixed microburners more stable, a preheating of their air is useful and can be obtained by using the air itself to cool the cap of the combustor. This is a good example of coupling 
two needs with one solution. In the present paper, the cooling of the cap is therefore studied in depth. On one hand, the whole thermal behavior of the combustor was analyzed, taking into account the wall radiative heating due to the radiation emitted from the hot gases within the combustion chamber. On the other hand, the impingement cooling on the cap was evaluated. Both analyses are coupled together, since the cap receives effects both from the impingement cooling and from the radiation coming from the whole combustor.

The use of the impingement cooling to cool combustor liners is quite common and is particularly important in lean premixed combustors as it allows to rise heat transfer coefficient using a limited amount of air in the combustion zone at the same time. Usage of this cooling technique in combustion chambers, also in conjunction with the effects of cooling on pollutant formation, is described in [1]. An experimental and numerical study of an impingement cooled combustion chamber was performed by Bailey et al. [2] who investigated heat transfer distribution on a combustor liner combining the use of impingement jet cooling with turbulators and variable passage geometry.

\section{NOMENCLATURE}

\begin{tabular}{|c|c|c|}
\hline$a_{\varepsilon, n}$ & Weighting factor & {$[-]$} \\
\hline$b_{\varepsilon, n, j}$ & Weighting factor & {$[-]$} \\
\hline$c$ & progress variable & {$[-]$} \\
\hline$D a, K a$ & Damkohler and Karlovitz number & {$[-]$} \\
\hline E & Emissive power & {$\left[W m^{-2}\right]$} \\
\hline$h$ & Heat transfer coefficient & {$\left[W m^{-2} k^{-1}\right]$} \\
\hline$I$ & Total radiative intensity & {$\left[W m^{-2}\right]$} \\
\hline$I_{\lambda}$ & Radiative spectral intensity & {$\left[W m^{-3}\right]$} \\
\hline$J$ & Wall radiosity & {$\left[W m^{-2}\right]$} \\
\hline$k$ & Turbulent kinetic energy & {$\left[m^{2} s-2\right]$} \\
\hline$L_{m}$ & Mean beam length & {$[m]$} \\
\hline$L_{n}$ & Beam segment length & {$[m]$} \\
\hline$L_{T}$ & integral turbulence length scale & {$[m]$} \\
\hline$N$ & Number of elements & {$[-]$} \\
\hline$p$ & Sum of partial pressures & {$[P a]$} \\
\hline$q$ & Heat flux & {$\left[W m^{-2}\right]$} \\
\hline$S^{k}$ & Energy equation source therm & {$[W]$} \\
\hline$S_{c c}$ & Combustion chamber liner surface area & {$\left[m^{2}\right]$} \\
\hline$S$ & Strain rate module & {$\left[s^{-1}\right]$} \\
\hline$S_{L}, S_{T}$ & Laminar and turbulent flame speed & {$\left[m s^{-1}\right]$} \\
\hline$s$ & Coordinate along direction $\vec{\Omega}$ & {$[m]$} \\
\hline$T$ & Temperature & {$[K]$} \\
\hline$T_{m}$ & Gas characteristic temperature & {$[K]$} \\
\hline$U_{i}$ & Mean Velocity component & {$\left[m s^{-1}\right]$} \\
\hline$\underline{V_{c c}}$ & Polynomial coefficient & {$\left[m^{3}\right]$} \\
\hline$\overline{v^{2}}$ & Turbulence scale velocity & {$\left[m^{2} s^{-2}\right]$} \\
\hline \multicolumn{3}{|l|}{ Greeks } \\
\hline$\delta_{l}$ & laminar flame thickness & {$[m]$} \\
\hline
\end{tabular}

$\begin{array}{lll}\varepsilon & \text { Turbulence dissipation } & {\left[\mathrm{m}^{2} \mathrm{~s}^{-3}\right]} \\ \varepsilon_{g} & \text { Gas total emissivity } & {[-]} \\ \varepsilon_{w} & \text { Wall emissivity } & {[-]} \\ \kappa_{a} & \text { Absorption coefficient } & {\left[\mathrm{m}^{-1}\right]} \\ \kappa_{\lambda} & \text { Spectral absorption coefficient } & {\left[\mathrm{m}^{-1}\right]} \\ \lambda & \text { Wavelength } & {[\mathrm{m}]} \\ \mu_{,} \mu_{T} & \text { molecular and turbulent viscosity } & {[\mathrm{kg} / \mathrm{m} / \mathrm{s}]} \\ \xi & \text { fuel mixture fraction } & {[-]} \\ \rho & \text { Density } & {\left[\mathrm{kgm}^{-3}\right]} \\ \rho_{w} & \text { Wall reflectivity } & {[-]} \\ \sigma_{\lambda} & \text { Spectral scattering coefficient } & {\left[\mathrm{m}^{-1}\right]} \\ \sigma & \text { Stefan-Boltzmann constant } & {\left[\mathrm{Wm}^{-2} \mathrm{~K}^{-4}\right]} \\ \tau & \text { Turbulence time scale } & {[\mathrm{s}]} \\ \Phi_{\lambda} & \text { Spectral scattering phase function } & {[-]} \\ \vec{\Omega} & \text { Propagation direction } & {[-]} \\ \Omega_{c} & \text { Progress variable source term } & {\left[\mathrm{kg} / \mathrm{m}^{3} / \mathrm{s}\right]}\end{array}$

\section{Superscripts \\ $k \quad$ Index \\ $o \quad$ Black body}

$\begin{array}{ll}\text { Subscripts } \\ B & \text { Beam } \\ b & \text { Burned } \\ c d & \text { Compressor discharge conditions } \\ g & \text { Gas } \\ i, j & \text { Index } \\ L & \text { Liner } \\ m & \text { Mean or characteristic value } \\ n & \text { Zone number } \\ P & \text { Patch } \\ T & \text { Turbulent } \\ u & \text { Unburned } \\ w & \text { Wall property }\end{array}$

\section{Computational Approach 1.1 Research Outline}

As mentioned above, the aim of the present work is to supply an effective computational support to the design activity of a new combustor and, in particular, to the development of the liner cap cooling system. The work is subdivided in various different steps which involve different parts of the combustion system. The different stages of the research can be summarized as follows:

- reactive flow computations, to define the flow field and temperature distribution within the combustor liner;

- estimation of the radiative heating on the hot side of the cap wall through computations taking into account the radiative behavior of flame and liner walls;

- analysis of the impingement heat transfer on the cold side of the cap; 
The last two points involve problems which are physically coupled as the heat flux actually passing through the cap wall depends on the thermal radiation emitted by the hot side of the cap (see $\S 2.2$ ). According to the Stefan-Boltzmann equation (see $\S$ 1.4), the radiation emitted by the wall depends on the temperature of the wall itself which is obviously affected by the cooling on the cold side.

As will be seen in more details at $\S 1.2$, the reactive/radiative computations and the analysis of the cooling system have been carried out using two different computational models; therefore, to correctly match the thermal boundary conditions on the cap wall for the two domains an iterative procedure was used. This procedure is summarized below.

- Initialization by means of a reactive/radiative computation on the combustor (see $\S 1.2$ ) in which the liner walls were assumed to be adiabatic (namely, the temperature is determined by the code through the condition that the resulting global heat flux passing the wall is zero);

- estimation of a first value of the cap wall temperature by carrying out a computation on the impingement cooling system model (see $\S 1.2$ ) imposing on the cap a heat flux equal to the thermal incident radiation coming out from the previous calculation;

- new radiative computation imposing the temperature determined in the above step as a new thermal boundary condition; this allows to compute a value of the cap radiosity and therefore a new value of the heat flux;

- the new heat flux value is again imposed on the impingement cooling model;

- the last two steps are repeated until the variation of the thermal boundary conditions on the cap wall (heat flux and wall temperature) among two subsequent runs are below a threshold value which is assumed to be acceptable (given the overall accuracy of the whole approach).

A matching is achieved after a few iterations of the previously described procedure. We finally notice that the effect of the wall thermal resistance may be assumed to be negligible. Actually, the liner walls are very thin and, as consequence, the temperature distribution through the wall thickness may be assumed to be uniform.

\subsection{Global Numerical Approach}

The present work is based on steady RANS computations. The computations were carried out using the 3.150 release of the STAR-CD CFD commercial package. As mentioned above, the analysis was performed using two different computational models: one for the combustion chamber and one for the cooling system. The thermal coupling between the two computations was obtained by means of the iterative procedure previously outlined.

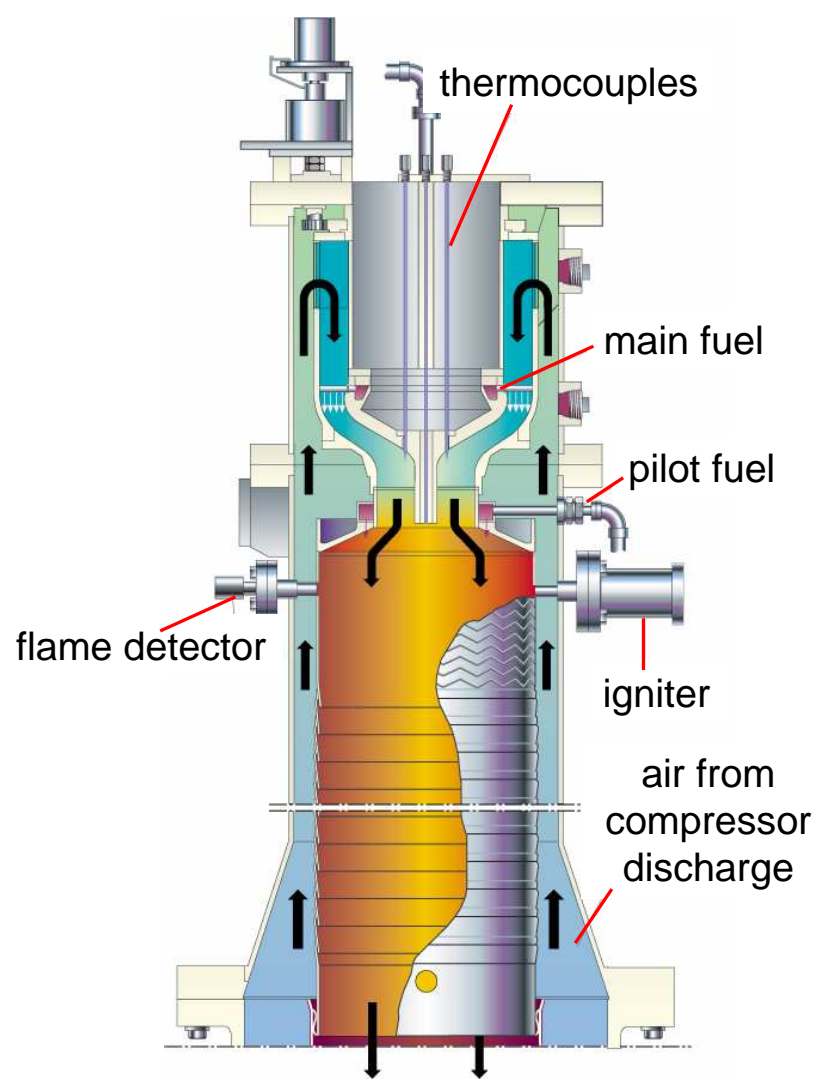

Figure 1. GE10 gas turbine combustion chamber

This approach was preferred to one single simulation of the whole system because of the difficulty in the geometrical matching of the two computational models (which will be made clear below), and also because of its greater flexibility (from the viewpoint of the overall computational procedure).

The combustion chamber is a single can combustor as shown in figure 1. The geometry of the upper part of the liner is characterized by 32 premixed microburners used as pilot burners (figure 2). As a consequence, the liner shows a geometrical periodicity of $1 / 32$ th of a full angle in the circumferential direction. A similar statistical periodicity of the flow field may then be assumed. Therefore, the computational domain reproduced just a $11.25^{\circ}\left(360^{\circ} / 32\right)$ wide sector of the whole combustion chamber; periodic boundary conditions were imposed on the meridional sections of the domain.

The main flow enters the combustor through a premixing duct (see figure 3). Any swirl component of the main flow is suppressed within the premixing duct by means of a bladed device (see figure 1); therefore, the main flow direction at the actual inflow section of the liner may be assumed to be axial.

The geometry of the swirled microburners (with its own pi- 


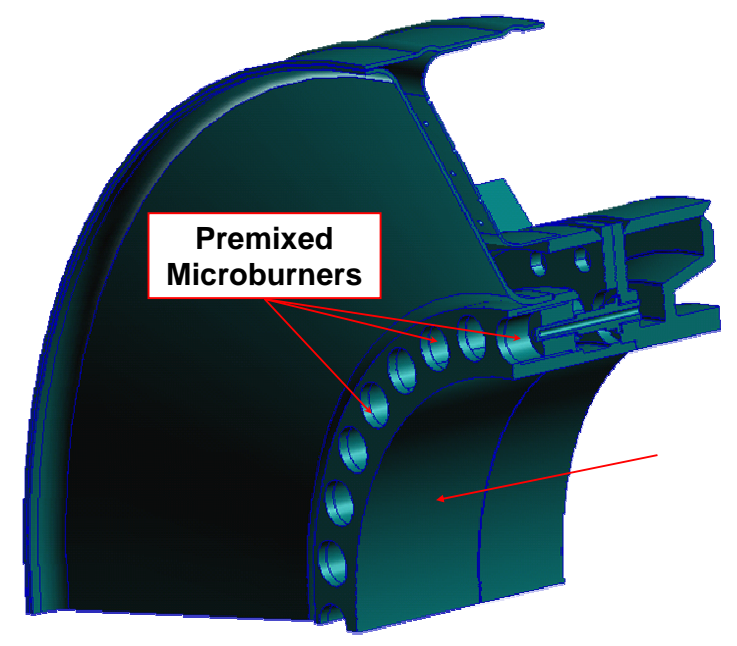

Figure 2. Details of the cooling system for the cap of the combustor.

lot fuel injection holes) is discretized through an appropriately refined mesh connected to the liner (see figure 3 and 4). The upper part of the liner (the "cap") has a conical shape in order to control the size of side-toroidal-recirculation-zone produced by the main flow sudden expansion into the combustion chamber. The side-recirculation-zone is the main promoter of the flame stabilization causing a direct exposure of the cap (highlighted in figure 3 ) to the high temperature region of the flow field.

The secondary air holes rows placed on the upper part of the side wall of the liner show a different periodicity (with respect to the overall geometry); each row was simulated by means of circumferential slots with an equivalent passage area as shown in figure 3 .

Care was taken to correctly distribute the whole air and fuel mass flow rates among the various inlets in the model, namely, the design mass flow rates were assigned to each inflow section of the model.

A finer computational grid was used within the liner in the region just downstream the microburner to accurately solve the flow field and the temperature distribution in this region. To make the computations not too expensive, the couple tool of the software package was used to coarsen the grid as you move downstream this high gradients region (figure 3). Nevertheless, the mesh complete connectivity was preserved whenever possible. The final mesh consists of about 1,100,000 cells. The development of the present computational grid was based on a study previously carried out on a very similar combustion chamber for which an accurate grid independence analysis was performed.

The code makes use of a face centered scheme for diffusive terms, while a second order advection-reconstruction scheme was chosen for the discretization of the convective terms.

A $k-\varepsilon$ high Reynolds turbulence model was used for

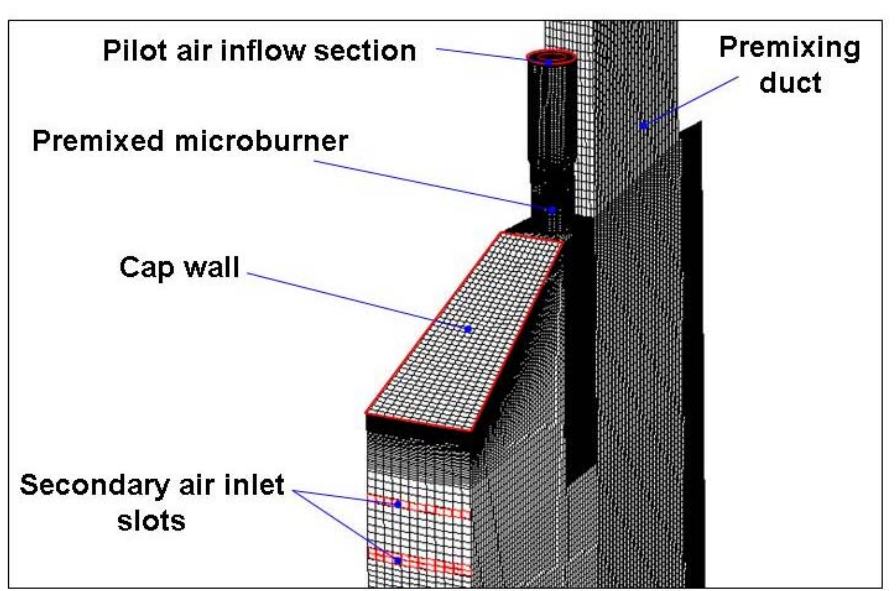

Figure 3. Detail of the computational grid for the reactive and radiative computations

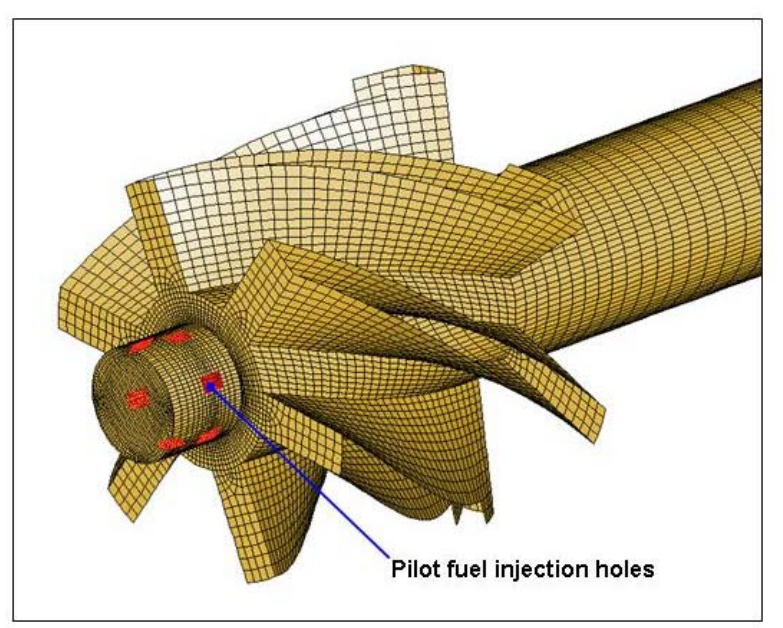

Figure 4. Details of the geometry and computational grid of the swirled microburner

the computations in the combustion chamber, while a BML (Bray-Moss-Libby) turbulent combustion closure based on Kolmogorov-Petrovskii-Piskunov theorem (see $\S 1.3$ for details) was used to model the combustion process.

Radiative computations were carried out by means of the native radiation model available in the software package which makes use of the discrete transfer method to solve the radiation transfer equation (see $\S 1.4$ for a description of the overall approach used in the code to model radiative heat transfer).

Velocity magnitude and direction, fluid temperature, turbulence intensity and dissipation length scale (as well as progress 


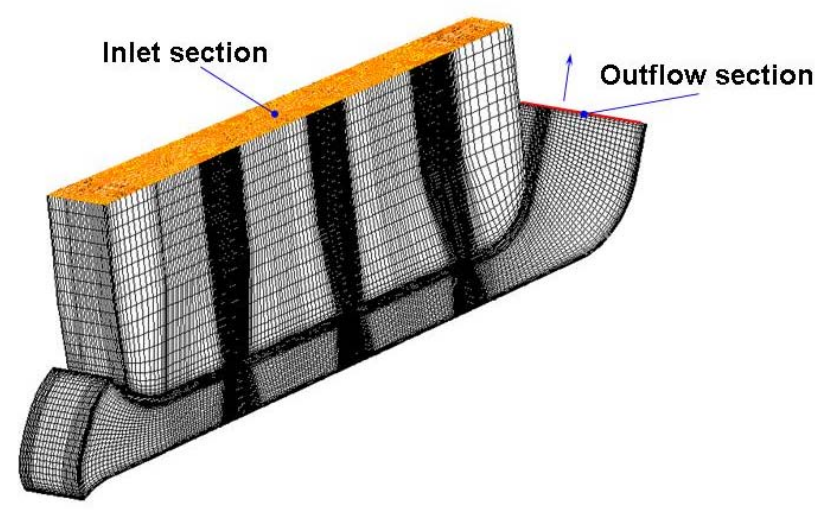

Figure 5. Computational grid for the cap cooling system. Inflow and outflow sections are shown.

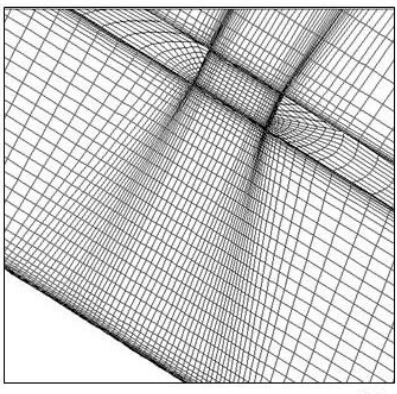

(a)

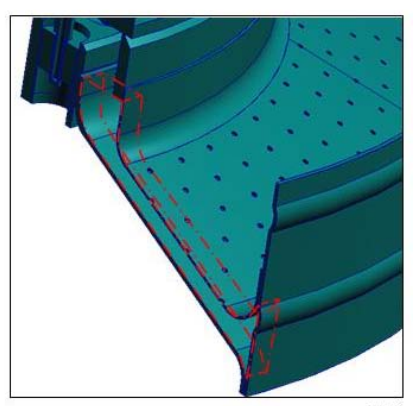

(b)
Figure 6. (a) Detail of the computational grid in the impingement jet region. (b) Modeled portion of the cooling system.

variable and mixture fraction) were specified at all the inflow sections of the model. A constant static pressure condition was specified as boundary condition at the discharge section.

The computational model used to study the cap cooling system is shown in figure 5. The impingement cooling is made of 68 lines of staggered jets (see figure 6 (a)). For the present case the minimum geometrical (and physical) periodic element is found to be a sector which width is 1/136th of the whole combustor (actually each holes row may be split along a meridian plane of the combustion chamber containing the row holes axis).

As pointed out above, the different periodicity between the two geometries (and therefore between the two computational models) makes it very difficult to directly couple the reactive/radiative computations with those to be carried out on the cooling system (the whole combustion chamber would have to be computed); therefore, two different numerical models were used.
The cooling system mesh counts 700,000 cells. It models just the minimum periodic element of the geometry; symmetry boundary conditions were imposed on both the meridian sections of the model. The mesh was built following some criteria established in previous works on similar flows, a correct value of the height of the first cell adjacent to the wall as well as an adequate number of cells in the boundary layer were ensured.

A fictitious upper plenum was added to simulate the chamber feeding the impingement holes. For the present computations a first order accurate upwind scheme was used for the discretization of the convective terms (in order to obtain a more stable computation).

Regarding the turbulence modeling, a realizable, high Reynolds, $k-\varepsilon$ turbulence model with a two layer formulation at the wall was used for the present case. The Durbin realizability condition (see [3]) was implemented in the code (by means of the user subroutine tools) to prevent the well known $k-\varepsilon$ stagnation point anomaly which leads to an overestimation of the heat transfer coefficient in the stagnation region. The Durbin realizability condition imposes a bound on the turbulence time scale according to:

$$
\tau=\min \left(\frac{k}{\varepsilon}, \frac{2 k}{3 \overline{v^{2}} C_{\mu}} \sqrt{\frac{3}{8\left|S^{2}\right|}}\right)
$$

where $\overline{v^{2}}$ is a turbulence scale velocity (coinciding with $k$ for the $k-\varepsilon$ model), and $S$ is the mean strain module

$$
S=\sqrt{S_{i j} S_{i j}}
$$

$$
S_{i j}=\frac{1}{2}\left(\frac{\partial U_{i}}{\partial x_{j}}+\frac{\partial U_{j}}{\partial x_{i}}\right)
$$

$U_{i}$ are the mean flow velocity components. A constant pressure boundary condition was chosen for the inflow as well as for the outflow sections of the model. The discharge pressure was gradually varied to match the design mass flow rate value through the model. Apart from the lower one, all the walls in the model were assumed to be adiabatic.

\subsection{Turbulent Combustion Model}

This approach is mainly based on the work of Schmid et al. [4] and of Lipatnikov and Chomiak [5]. The original model was modified in order to take into account the inhomogeneous distribution of inlet mixture fraction, characterized by an imperfect premixing and by pilot fuel jets, following, in some manner, the approach used by Polifke et al. [6].

The model is based on the classical approach of Bray-MossLibby (BML) [7] where a conservation equation for the time averaged progress variable $c$ is solved: 


$$
\frac{\partial}{\partial x_{i}}(\bar{\rho} \tilde{c})+\frac{\partial}{\partial x_{i}}\left(\bar{\rho} \tilde{c} \tilde{u}_{i}\right)=-\frac{\partial}{\partial x_{i}}\left(\bar{\rho} \widetilde{c}^{\prime \prime} u_{i}^{\prime \prime}\right)+\tilde{\Omega}_{c}
$$

In this work a standard gradient diffusion assumption was made for scalar flux:

$$
\bar{\rho} \overline{c^{\prime \prime} u^{\prime \prime}}=-\frac{\mu_{T}}{S c_{T}} \frac{\partial \tilde{c}}{\partial x_{i}}
$$

As known, this approach is often criticized due to the tendency to counter gradient behavior for scalar flux [7]. Following a recent review paper by Veynante ad Vervisch [8], where a detailed analysis of gradient (GD) and counter gradient diffusion (CGD) is made on the base of Direct Numerical Simulation (DNS) data, it's possible to locate CGD in the region of wrinkled flamelet on the so called Borghi diagram (where $D a>>1$ and $K a<<1$, see [7]): it's known that lean premixed combustors operates at Damkholer number close to 1 in the so called Thin Reaction Zone [6]. However, it's not possible to exclude that local effects of counter gradient diffusion influence scalar flux, especially in the low turbulence zone of the flame [5], but the approximation of complete GD is assumed to be sufficiently accurate for the present work.

The source term for equation 4 is evaluated via the usual dependency of $\Omega_{c}$ on $c$, in the form $c(1-c)$; recalling the solution of the Kolmogorov-Petrovskii-Piskunov theorem (KPP) [5], Schmid et al. proposed:

$$
\tilde{\Omega}_{c}=\bar{\rho}_{u} C_{K P P} \frac{S_{T}^{2}}{D^{T}} \tilde{c}(1-\tilde{c})
$$

where $C_{K P P}$ is a model constant, $D_{T}$ is the turbulent diffusivity and $S_{T}$ is the turbulent burning velocity. To evaluate $S_{T}$ the following phenomenological approximation is used ( [4], [5]):

$$
S_{T}=S_{L}^{0}+u^{\prime} \cdot\left(1+D a^{-2}\right)^{\frac{1}{4}}
$$

where Damkohler number is evaluated as:

$$
D a=\frac{\tau_{T}}{\tau_{L}} \approx \frac{L_{T}}{u^{\prime}} \cdot \frac{S_{L}^{0}}{\delta_{L}}
$$

with:

$$
L_{T}=C_{\mu} \frac{k^{3 / 2}}{\varepsilon}
$$

being $\delta_{L}$ the thickness of laminar premixed flame.

In order to take into account the effects of imperfect premixing of reactant mixture, mainly due to the injection of pure fuel through pilot jets, an additional conservation equation for the averaged mixture fraction $\xi$ was solved:

$$
\frac{\partial}{\partial t}(\bar{\rho} \tilde{\xi})+\frac{\partial}{\partial x_{i}}(\bar{\rho} \tilde{\xi} \tilde{u})=-\frac{\partial}{\partial x_{i}}\left(\frac{\mu_{T}}{S c_{T}} \frac{\partial \tilde{\xi}}{\partial x_{i}}\right)
$$

The dependency of gas temperature and density (both burned and unburned) and of laminar flame velocity and thickness on mixture fraction, was evaluated following the approximation already used in other works ( [6], [9]). In particular, calling $Q$ the generic scalar, we assume $\overline{Q(\xi)}=Q(\bar{\xi})$. This way of modeling neglects the effects of turbulence fluctuations on fuel concentration and therefore its influence on combustion process that may be relevant in industrial burners, especially near extinction limits. To deal with such effects, the introduction of mixture fraction variance and, usually, the assumption of its probability distribution, are required but this type of approach will not be used here, in order not to alter the simplicity of the model. Mixture fraction dependency of temperature, density and main species (reactants, fuel and main products) was evaluated with equilibrium chemistry calculations, while the curves $S_{L}(\xi)$ and $\delta_{L}(\xi)$ were calculated with the correlations of ([10]): data were collected in an external look up table. Local averaged temperature and density were finally calculated as follows:

$$
\tilde{Q}=(1-\tilde{c}) \cdot \tilde{Q}_{u}+\tilde{c} \cdot \tilde{Q}_{b}
$$

\subsection{Radiation Model}

Because of the high temperatures achieved in the combustion chamber the radiative heat transfer inside the burner cannot be neglected; on the contrary, as will be seen in $\S 2.2$, it constitutes the main heating source for the liner walls. Therefore, an analysis of the radiative interaction between the liner surfaces and the gas within the chamber is crucial for a reliable prediction of the cooling system performance.

As mentioned above, the radiative computations were carried out based on the native radiation model available in the STAR-CD software package. In the present section we will give a short account of the theoretical background of radiative heat transfer with a participating gas, and a brief outline of the main features of the model (see [11], [12] and [13] for further details).

Accurate calculations of radiative heat transfer are very complex, mainly because of the strong spectral dependence of the radiative properties of the combustion products. In natural gas combustion, among the species present inside the liner, only $\mathrm{CO}_{2}$ and $\mathrm{H}_{2} \mathrm{O}$ show a significant interaction with thermal electromagnetic waves, while $\mathrm{CH}_{4}$ and $\mathrm{CO}$ are of minor importance in this respect ([12], [14]).

The foundation of almost all the radiative transfer analyses at an engineering level is the radiative transfer equation (RTE). 
The RTE is a simplification of the full system of the Maxwell equations. It does not account for polarization effects and for the influence of nearby particles on scattering phenomena; it's assumed to be valid for most of the engineering applications. For a steady state transfer the RTE can be written as follows:

$$
\begin{aligned}
\frac{d I_{\lambda}(s, \vec{\Omega})}{d s}+\kappa_{\lambda} I_{\lambda}(s, \vec{\Omega})+\sigma_{\lambda} I_{\lambda}(s, \vec{\Omega})=\kappa_{\lambda} I_{\lambda}^{o}(T(s))+ \\
+\frac{1}{4 \pi} \int_{4 \pi} \sigma_{\lambda} \Phi_{\lambda}\left(\vec{\Omega}, \vec{\Omega}^{\prime}\right) I_{\lambda}\left(s, \vec{\Omega}^{\prime}\right) d \vec{\Omega}^{\prime}
\end{aligned}
$$

This equation expresses the conservation of radiant energy. The first term on the left hand side is the change in spectral radiant intensity along the path length $d s$ in the direction of vector $\vec{\Omega}$ at the location $s$. This is due to:

- absorption of radiant energy (second term on the left hand side);

- out scattering (third term on the left hand side);

- energy emission from the gas along the path length $d s$ (first term on the right hand side);

- in scattering from the outside in direction $\vec{\Omega}$ (integral term).

The equation is written in this form to allow for accounting for the spectral variation of properties of the medium. Various models have been developed to handle the complex spectral structure of the radiative properties of the gases. This task happens to be quite difficult at least with simple models, while more complex and physically sound models usually involve a great increment in the computational resources needed to carry out the radiative calculations. Much of the work at an engineering level in this field is therefore performed by assuming a simple gray gas model (SGG) for the spectral behavior of the medium. Namely, the spectral properties of the gas are assumed to be constant over the whole wavelength spectrum ([12]). Equation 12 may then be integrated with respect to the wavelength to obtain an equation for the total radiation intensity:

$$
I(s, \vec{\Omega})=\int_{0}^{\infty} I_{\lambda}(s, \vec{\Omega}) d \lambda
$$

Moreover, for gaseous fuels the scattering phenomena in the mixture are usually negligible (while being significant in the combustion of liquid fuels because of the formation of soot particles) therefore, for the scattering coefficient we can put $\sigma_{\lambda}=0 \mathrm{~m}^{-1}$ over the whole spectrum.

With the above assumption the RTE can be written as:

$$
\frac{d I(s, \vec{\Omega})}{d s}=\kappa_{a}\left(I^{o}(T(s))-I(s, \vec{\Omega})\right)
$$

For the black body radiation we have $I^{o}(T(s))=\sigma T(s)^{4} / \pi$.

Various techniques have been developed to integrate this equation. The technique implemented in the STAR-CD code is

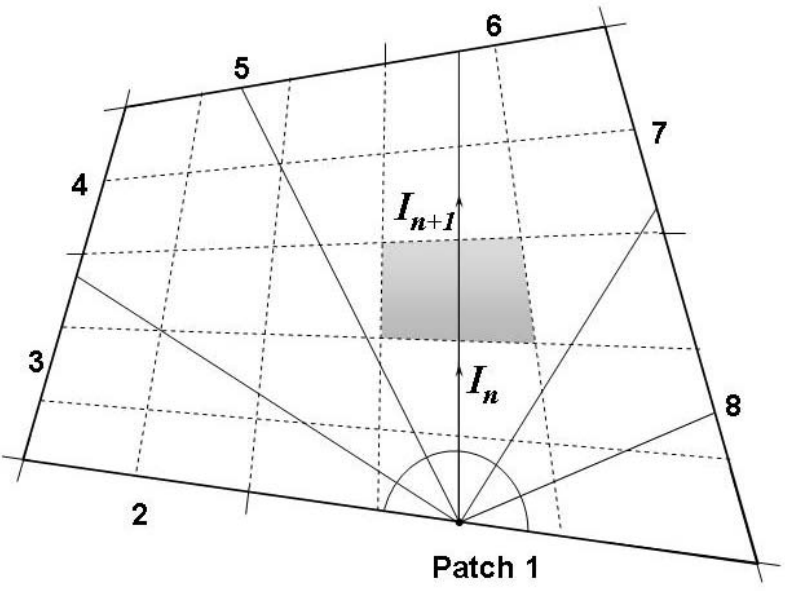

Figure 7. Radiation modeling computational approach.

the discrete transfer method (DTM, [11]). In this method equation 14 is discretized along ray beams; the path along a beam is discretized on the segments individuated by the intersection of the beam with the faces of a cell of the computational domain (see figure 7). Assuming the physical properties to remain constant along the length of the ray inside the cell, equation 14 may be integrated analytically to yield:

$$
I_{n+1}=I_{n} e^{-s_{n}}+\frac{L_{n}}{s_{n}} \frac{\kappa_{a}}{\pi} \sigma T_{n}^{4}\left(1-e^{-s_{n}}\right)
$$

where $I_{n}$ and $I_{n+1}$ are the beam intensities entering and leaving the cell respectively, $L_{n}$ is the length of the beam segment inside the cell, and for $s_{n}$ we have $s_{n}=\kappa_{a} L_{n}$.

The rays on which the equation is discretized are traced from the nominal center of the radiative "patches" into which the surface boundaries of the computational domain are subdivided. The patches are used to discretize the surfaces of the domain in order to take into account their radiative properties (see below for some additional details and [13] for a complete description of the STAR-CD methodology for radiative computations).

Since there is an energy exchange between the radiant beam and the media, an additional source term needs to be put in the enthalpy equation. If we consider the beam $i$, of (solid) angular width $\delta \vec{\Omega}_{i}$ coming from patch $j$, the contribution to the energy density of cell $k$ is:

$$
S_{i j}^{k}=\left(I_{n+1}-I_{n}\right) \vec{\Omega}_{i} \cdot d \vec{A}_{j} \delta \vec{\Omega}_{i}
$$

where $d \vec{A}_{j}$ is the surface area of the emitting patch. The total source term due to all the beams passing through cell is therefore: 


$$
S^{k}=\sum_{N_{P}} \sum_{N_{k j}} S_{i j}^{k}
$$

Where $N_{P}$ and $N_{i j}$ are the total number of patches in the model and the number of beams from patch $j$ entering the cell $k$. In the present computations this contribution to the energy equation is not accounted for since the combustion model does not explicitly solve an energy equation.

As pointed out above, the boundaries of the computational domain (including the inflow and outflow boundaries but not symmetry and periodic ones) need to be subdivided into a number of contiguous non overlapping radiative patches. From the center of each patch a number of radiant beams is traced to intercept opposite patches. The number $\left(N_{P}\right)$ and distribution of the patches on the boundaries as well as the number of the beams $\left(N_{B}\right)$ originating from each patch are chosen by the user. The thickness of the beams (width of the subtended solid angle) is uniform over the beams departing from a patch, and the ensemble of the beams covers the whole unit hemisphere centered in the centroid of each single patch (see figure 7).

The traced ray beams are used both to compute the view factors between the patches and to solve the RTE as previously shown, if a participating media is present. In such a way the radiant energy transfer between each couple of viewing patches and, eventually, the participating media, could be computed.

The number and distribution of the patches and the specified number of beams per patch have to be selected as a tradeoff between accuracy and computational cost. In fact, the distribution of the patches has to adequately represent the geometry, and the number of beams have to be selected so that they allow a reliable evaluation of how the various parts of the domain "see" each other (in fact a low number of beams reduces the likelihood that the other patches are intercepted by a beam drawn from a patch, see again figure 7) and has to guarantee a good uniformity of heat flux and temperature over each patch, to avoid the introduction of numerical errors in the computations.

Fluid boundaries (inflow, outflow and eventually free stream boundaries) are treated as "escape" boundaries assuming they behave as black bodies. Their radiative intesity is defined by assigning a specified boundary temperature. All the surfaces are assumed to be of the gray Lambert type and to give diffuse reflection.

\section{Results}

In this section we will analyze the results of the various stages of the work. First of all a brief description of the reactive computations will be given: the main details of the velocity and temperature fields will be expounded. Then, the outcome of the radiative computations will be examined and the criterion used to appropriately select the radiative properties of the gas mixture will be shown in detail. Finally, we will describe the results obtained from the computations performed on the cooling system, and a comparison with some experimental measurements of the cap wall temperature will be given.

\subsection{Flow Field and Temperature Distribution}

In figure 9 and 10 the distribution of the axial component of the velocity is shown. Figure 9 shows the formation of a central recirculation zone just downstream of the microburner due to the action of the swirler embodied in the burner. The pilot flame is therefore stabilized in this zone ensuring the anchorage of the main flame. A side recirculation zone is generated in the side zone of the combustion chamber because of the sudden expansion of the liner section (figure 10).

The axial extent of this region is quite long and the velocity magnitude is very little as could be seen in figure 8 . This flow structure gives rise to a very long main flame as shown in figure 11 (where the cooling effect of the secondary air injection in the upper portion of the liner is also shown). The pilot flame has a "twisted" shape because of the tangential velocity component (with respect to the microburner axis) imposed on the flow field by the swirler (figure 12).

Concerning the temperature distribution we wish to point out that the fast chemistry assumption of the turbulent combustion model causes a slight overestimation of the peak flame temperature (about $2400 \mathrm{k}$ ), which, in the present case, can be found in the stoichiometric region just downstream the microburner.

\subsection{Radiative Computations}

As described in $\oint 1.4$, setting up a radiative calculation in the STAR-CD CFD package requires the definition of an adequate number and distribution of patches and the selection of a consistent number of beams per patch. As a preparatory work for the present study, a preliminary sensitivity analysis to these parameters was performed on a simplified model. Then, on the basis of this test, and taking into account that the zone of interest for the present work is mainly the upper part of the combustion chamber, proper values for $N_{P}$ and $N_{B}$ could be selected. Many of the patches were located in the upper part of the domain, while fewer and fewer patches were used going down along the axis of the combustor, towards the outflow section.

Concerning the boundary conditions for the RTE, the following assumptions were adopted. The liner walls were assumed to behave as black surfaces. The selection of a proper value for the emissivity of the combustion chamber walls is somewhat arbitrary as it depends on the material, the temperature and the degree of oxidation of the surface. Moreover, it changes during the life of the liner because of oxidation and deposition of particulates. Assuming $\varepsilon_{w}=1$ is a conservative choice, at least from 


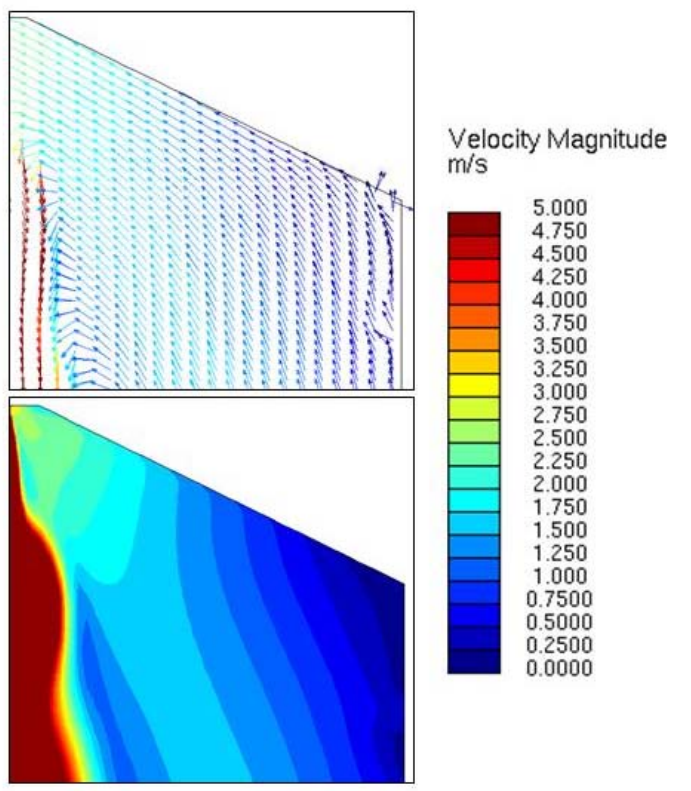

Figure 8. Velocity magnitude and vectors in the region just under the cap.

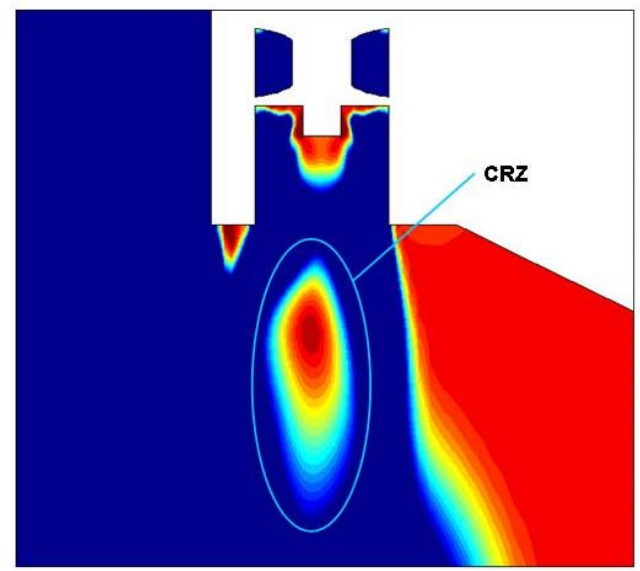

COMPONENT W M/S
LOCAL MX $=28.85$ LOCAL MN $=-90.36$
LOC

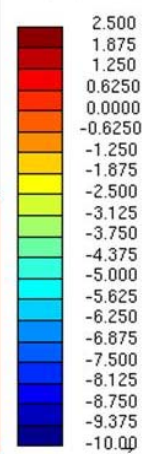

Figure 9. Velocity axial component: detail of the central recirculation zone

the thermal viewpoint, as it leads to higher wall temperatures; therefore, it seems to be adequate to the purpose of the present work.

As mentioned above ( $(2.2)$, inflow and outflow sections are treated as "escape" surfaces for the radiative beams: they absorb the whole radiation coming from the inside of the combustor. The incoming radiation is computed according to the StefanBoltzmann law on the basis of a temperature which is thought to

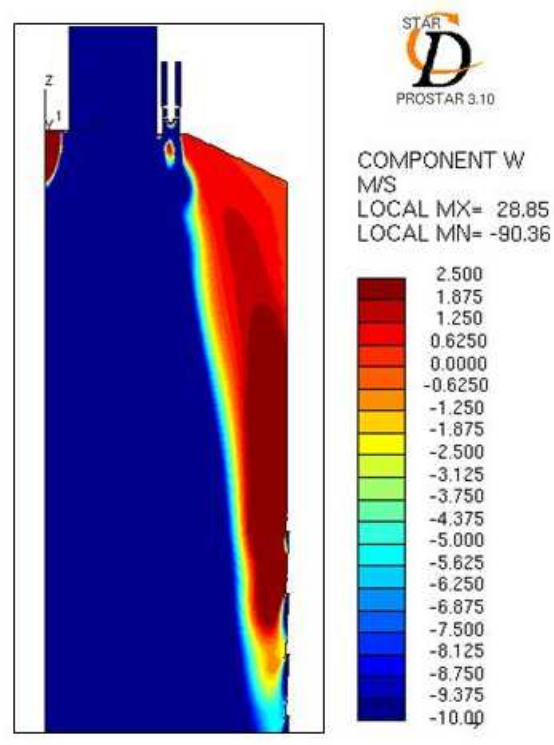

Figure 10. Velocity axial component: corner recirculation zone

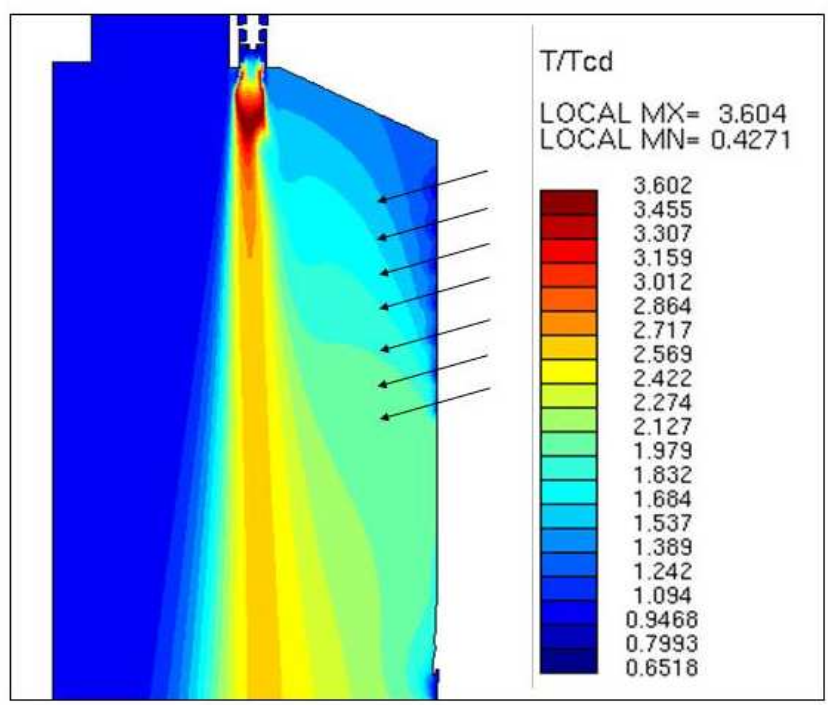

Figure 11. Temperature distribution in the upper part of the combustion chamber. The temperature is normalized dividing for the compressor discharge temperature. The effect of the secondary air injection is also shown.

be "representative" of the "ambient" temperature in the environments connected to the computational domain. The compressor discharge temperature $\left(T_{c d}\right)$ was imposed as "radiative" temperature on all the air inflow sections in the model. Ambient temperature (about $293 K$ ) was used for pure fuel inlets. For the discharge section of the combustion chamber, the average temperature on 


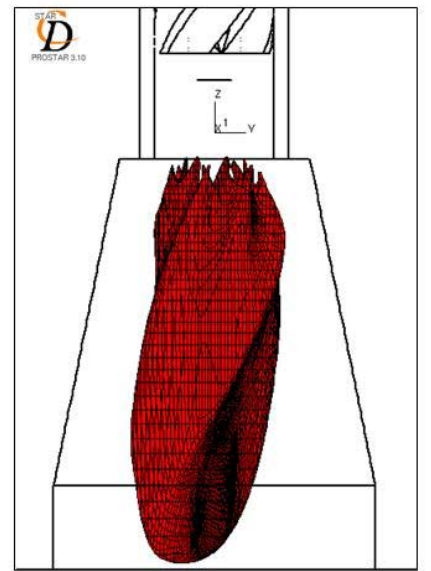

Figure 12. Progress variable iso-surface $(\tilde{c}=0.75)$ : twisted flame structure.

the outlet boundary obtained from the reactive non-radiative calculations was selected (see $\S 1.4$ for a discussion about radiative surface treatment in STAR-CD).

To complete the definition of the radiative computations, the selection of an appropriate value for the absorption coefficient $\kappa_{a}$ is needed. This is quite a difficult task because of the strong dependence of the radiative properties of the chemical species which compose the mixture on the frequency of the radiation. This makes the simulation of the radiative behavior of the mixture through a single parameter inaccurate to some extent. Generally speaking, the distribution of the heat flux as well as the temperature pattern on the heated surface will show a physically sound qualitative behavior (as can be seen in figure 13), but will be hardly accurately captured using this kind of model ([15]). Moreover, the definition of an appropriate value for $\kappa_{a}$ is somewhat arbitrary, as it can be assessed only by means of some kind of trial and error procedure. Following Liu et al. ( [15]), we can define the optimal value of the absorption coefficient as the value of $\kappa_{a}$ which ensures agreement on the total heat flux passing through the cap wall when the gas temperature field is specified. This definition implies the availability of an auxiliary procedure or method in order to evaluate, in a completely independent way, the heat sinking the wall.

For the present work this additional information was obtained by means of a correlative procedure for the heating of the cap wall developed at GE-Energy, through which the average heat flux on the cap of the liner was estimated. The computed radiative heat flux through the cap wall was compared with this value, and an evaluation of the reliability of the value selected for the $\kappa_{a}$ parameter could be performed.

Nonetheless, as mentioned above, the finding of an optimal value for $\kappa_{a}$, even if ensures that the energy balance is correctly respected, does not allow for an accurate prediction of the distributions of thermal quantities on the walls (heat flux, wall temperature, HTC...); therefore, it could be wise to use this kind of computations preferably to analyze the thermal behavior of the system in the mean, rather than locally.

An a priori estimate of the optimal value of $\kappa_{a}$ may be obtained through the knowledge of the total emissivity $\varepsilon_{g}$ of the gas on the whole liner surface. $\kappa_{a}$ is related to the total emissivity according to the following equation [15]:

$$
\kappa_{a}=-\left(1 / L_{m}\right) \ln \left[1-\varepsilon_{g}\left(T_{m}, L_{m}\right)\right]
$$

where $L_{m}$ and $T_{m}$ are a mean beam length and a characteristic gas temperature. In the present work the mean beam length was estimated according to ([14], [16]):

$$
L_{m}=3.6 \frac{V_{c c}}{S_{c c}}
$$

Where $V_{c c}$ and $S_{c c}$ are the volume and the surface of the combustion chamber respectively. For the present case $L_{m} \simeq 0.9 D_{L}$. Still following Liu et al. ( [15]), $\varepsilon_{g}$ was estimated by means of the exponential wide band model $(E W B)$ :

$$
\varepsilon_{g}=\sum_{n=1}^{N} a_{\varepsilon, n}\left(T_{g}\right)\left[1-e^{-k_{n} p L}\right]
$$

where $p$ is the sum of the partial pressures of the radiation sensitive species $\left(\mathrm{H}_{2} \mathrm{O}\right.$ and $\mathrm{CO}_{2}$ for the present case), $\mathrm{L}$ is the path length, $\kappa_{n}$ is a fitting coefficient which can be regarded as an optimal mean absorption coefficient (see [17]), and, finally, $a_{\varepsilon, n}$ are the weighting coefficients of the model computed through polynomial expressions:

$$
a_{\varepsilon, n}\left(T_{g}\right)=\sum_{n=1}^{N} b_{\varepsilon, n, j} T_{g}^{j-1}
$$

The weighting factors have to satisfy the following condition:

$$
\sum_{n=1}^{N} a_{\varepsilon, n}\left(T_{g}\right)=1
$$

The $b_{\varepsilon, n, j}$ coefficient are constant for a given set of gray gases. For the present case, using for $T_{g}$ the average temperature in the first third of the combustion chamber, a value of 1.3 $m^{-1}$ was found for $\kappa_{a}$. The thermal incident radiation distribution on the liner cap resulting from the computation is shown in figure 13 :

The radiative heat flux through the wall is obtained as the difference between the incident radiation $I$ and the wall radiosity $J$ :

$$
q_{i}=I_{i}-J_{i}
$$




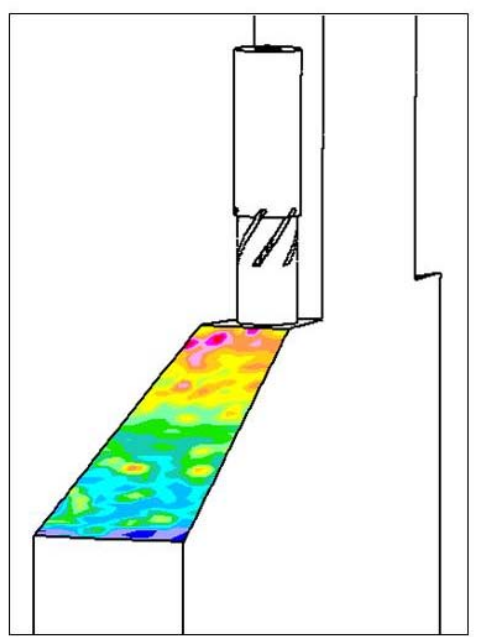

Thermal Incident Radiation $\left(\mathrm{W} / \mathrm{m}^{2}\right)$

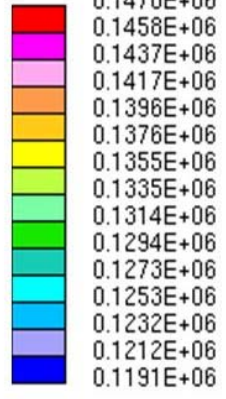

Figure 13. Distribution of the thermal incident radion on the liner cap

Here $q_{i}, I_{i}$ and $J_{i}$ are the radiative heat flux, the incident radiation and the radiosity on patch $i$. The radiosity of the wall is the sum of the emitted and reflected radiation, namely:

$$
J_{i}=\varepsilon_{w} E_{i}^{o}+\rho_{w} I_{i}
$$

Where:

$$
E_{i}^{o}=\sigma T_{w i}^{4}
$$

is the black body emissive power on patch $i$ according to Stefan-Boltzmann equation. From equation 25 it is seen that for the estimation of the radiative emission from the cap, knowledge of the wall temperature is needed. This establishes a physical coupling between the two computations as already examined above. As pointed out in $\S 1.1$, this coupling was rendered here through an iterative procedure which allowed to define physically consistent thermal boundary conditions to be imposed on the cap cooled wall. After a few steps of this iterative procedure, an appropriate wall temperature for the "hot side" (the normalized value of this temperature is $T_{w}^{\prime}=0.23660$, see the next section for the normalization procedure), and a coherent wall heat flux for the "cold side" $\left(q=125,000 \mathrm{Wm}^{-2}\right)$ could be defined.

As a final comment we notice that, as the velocity magnitude of the flow field in the region just under the cap are very low (see previous section and especially refer to figure 8), the heat transfer associated with convection is expected to be very little. This was also confirmed by a correlative evaluation of the convective HTC performed at GE-Energy. Therefore, the convective heat flux was assumed to be negligible with respect to the radiative heating, and the whole heat flux through the cap wall to be equal to the radiative heating alone.
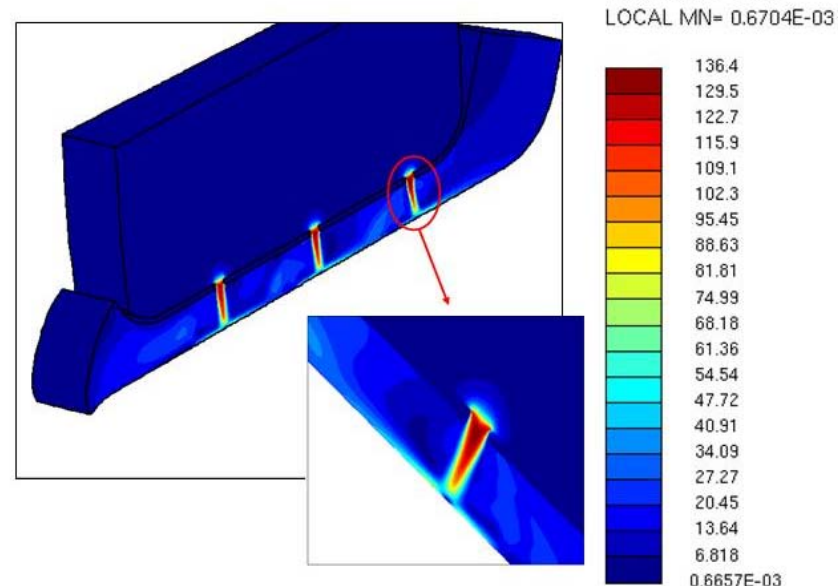

Figure 14. Velocity magnitude in the impingement cooling system. A detail of the flow structure in the proximity of the jet closer to the combustor axis is also shown.

\subsection{Cooling System Analysis}

Let's come now to the examination of the results obtained for the cooling system. First the flow field will be briefly examined, then we will analyze the thermal perfomance of the system.

In figure 14 the velocity magnitude distribution is shown. The crossflow develops in the centripetal direction as the impingement chamber is closed by the side wall of the liner. The ratio between the height of the impingement chamber and the diameter of the jet $(H / D)$ is quite high (about 3.2), therefore the crossflow velocity values stay low even in the zone closer to the axis of the combustor (even though the "duct" seen by the crossflow is convergent). Therefore, a weak crossflow is present within the impingement chamber and all the jets are characterized by high blowing rates. As a consequence, the jet's deflection is very little also for the last jets, as can be seen in figure 14 .

In figures 15 and 16 the distributions of the wall temperature and the heat transfer coefficient (HTC) are shown. The heat transfer coefficient was computed according to equation:

$$
h=\frac{q}{T_{w}-T_{c d}}
$$

As expected from the above analysis of the flow field, the minimum wall temperatures, and therefore the higher heat transfer coefficient (HTC) values are achieved just under the jets. While the higher temperatures are found in the peripheral zone (the most far away from the combustor axis, see also figure 17), where a dead water zone with very low velocities develops. Also, the temperature increase going towards the outflow section of the model as the cooling effect of the impingement jets gets weaker and weaker.

Temperature measurements on the cap wall were obtained by means of thermocouples located on cold side of the cap as 


\section{Normalized Wall \\ Temperature}

$T_{w}^{\prime}=\frac{T_{w}-T_{c d}}{T_{w}-T_{r e f}}$

\begin{tabular}{|l}
0.2160 \\
0.2080 \\
0.1999 \\
0.1919 \\
0.1838 \\
0.1758 \\
\hline 0.1677 \\
0.1596 \\
0.1516 \\
0.1436 \\
\hline 0.1355 \\
0.1275 \\
0.1194 \\
0.1113 \\
\hline 0.1033 \\
\hline $0.9525 \mathrm{E}-01$ \\
$0.8720 \mathrm{E}-01$ \\
$0.7915 \mathrm{E}-01$ \\
$0.7110 \mathrm{E}-01$ \\
$0.6305 \mathrm{E}-01$ \\
$0.5500 \mathrm{E}-01$
\end{tabular}

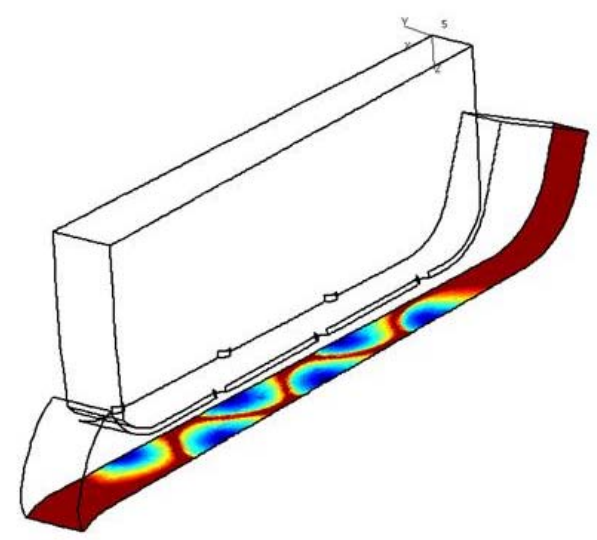

Finı ıre 15 Wall temneratı ure dictrihıtion $n$ n the liner can

\section{Normalized Heat}

Transfer Coefficient

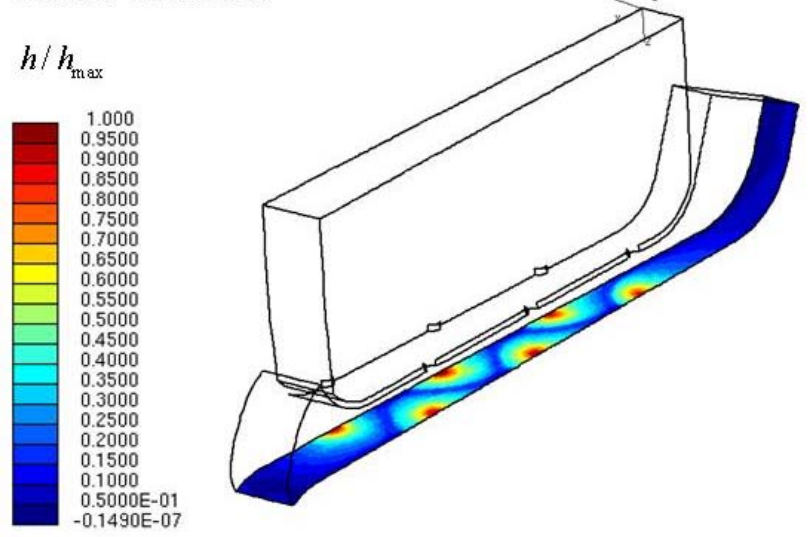

Figure 16. Heat transfer coefficient distribution on the liner cap

shown in figure 17. The temperatures measured by the thermocouples are shown in table 1, where a direct comparison with the spanwise averaged CFD results is shown. Wall temperature values were normalized according to equation:

$$
T_{w}^{\prime}=\frac{T_{w}-T_{c d}}{T_{w}-T_{r e f}}
$$

where $T_{c d}$ is the compressor discharge temperature and $T_{r e f}$ is an arbitrary reference temperature.

In figure 17 approximate radial locations of the thermocouples on a portion of the computational model lower wall are also shown: the computed local temperature values are just a little higher than the experimental but globally a good agreement is

\section{Normalized Wall Temperature}

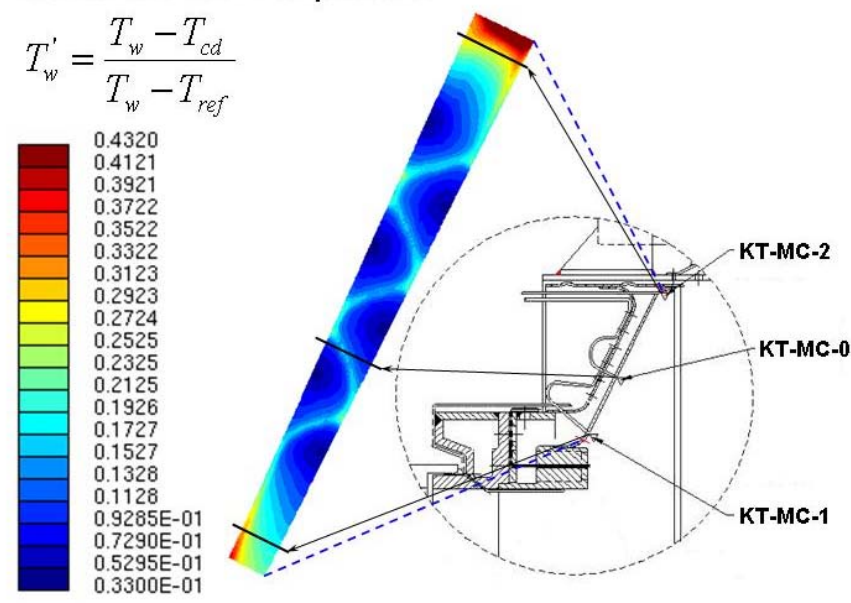

Figure 17. Normalized wall temperature distribution and thermocouple locations on the cap.

found.

\begin{tabular}{|c|c||c|c||c|c|}
\hline & $T_{w}^{\prime}$ & & $T_{w}^{\prime}$ & & $T_{w}^{\prime}$ \\
\hline \hline KT-MC-0 & 0.127 & KT-MC-1 & 0.190 & KT-MC-2 & 0.226 \\
\hline CFD & 0.154 & CFD & 0.223 & CFD & 0.276 \\
\hline
\end{tabular}

Table 1. Normalized wall temperatures measured on the liner cap and the correspondent spanwise averaged CFD results.

A mean value temperature was obtained performing an arithmetic average on the above data. The resulting normalized mean value is 0.18220 . The area weighted average on the whole lower surface is 0.23660 , but, if an appropriate portion of the cooled surface (individuated by excluding the peripheral parts where no temperature data is available, see figure 17), a value of about 0.19490 is obtained showing a very good agreement with the experimental data, which is also confirmed by the detailed comparison of table 1 .

\section{Concluding Remarks}

A numerical study of the heat transfer features of a single can combustion chamber was performed. The whole thermal behavior of the combustor was analyzed taking into account the wall radiative heating due to the radiation emitted from the hot gases within the combustion chamber.

One of the most critical zones is the upper part of the liner (here named "cap"), where the thermal load is expected to be 
very high because of the proximity of the pilot flame which is responsible for a strong radiative heating on the cap wall.

To maintain the temperatures of the liner walls in this region within admissible values, an impingement cooling system was integrated in the upper part of the combustion chamber. The heat transfer features of this zone of the combustor are quite complex and required a focused study. Therefore, two different numerical analysis for each one of the two parts of the system (the combustor itself and the cap cooling system) were carried out.

A study of the reactive flow field inside the combustion chamber was performed; then, the radiative heat transfer towards the cap wall was estimated by means of the native radiative model of the STAR-CD CFD package, which uses a discrete transfer method to integrate the radiative transfer equation, and a simple gray gas model to describe the radiative behavior of the gas mixture within the liner.

The radiative properties of the gray gas were estimated using a procedure based on a polynomial fitting of the spectral behavior of each gas. The reliability of the estimated value was tested comparing the calculated heat flux through the cap wall with the one resulting from a correlative procedure developed at GE-Energy. Then, computations on the impingement cooling system were performed.

Since the two models share a wall, a thermal coupling is clearly established. The coupling between the two numerical simulations was obtained through an iterative procedure through which the thermal boundary conditions on the two models are gradually lead to a matching.

The temperature distribution on the cap wall was finally obtained and compared with experimental measurements taken from the combustor liner at GE-Energy. A good agreement was found for the mean wall temperature as well as for the local values. The outcome of the present work turned out to be extremely useful in the following stages of the design process at GE-Energy.

\section{References}

[1] Schulz, A. "Combustor liner cooling technology in scope of reduced pollutant formation and rising thermal efficiencies". Annals New York Academy of Sciences, pp. 135-145.

[2] Bailey, J.C., Intile, J., Topaldi, A.K., Fric, T.F., Nirmalan, N.V., Bunker, R.S., 2002. "Experimental and numerical study of heat transfer in a gas turbine combustor liner". ASME Paper GT-2002-30183, pp. 1-12.

[3] Durbin, P. A., 1996. "On the k - $\varepsilon$ stagnation point anomaly”. Int. J. Heat and Fluid Flow, 17 , pp. 89-90.

[4] H.P., Schmid, P. Habisreuther, W., Leuckel, 1998. "A model for calculating heat release in premixed turbulent combustion flames". Combustion and Flame, 113 , pp. 79-91.

[5] Lipatnikov, A.N., Chomiak, J., 2002. "Turbulent flame speed and thickness: Phenomenology, evaluation and ap- plication in multidimensional simulations". Progress in Energy and Combustion Science, 28 , pp. 1-74.

[6] Polifke, W., Flohr, P., Brandt., M., 2000. "Modeling of inhomogeneously premixed combustion with an extended tfc model". ASME Paper 2000-GT-135.

[7] Poinsot, T., Veynante, D., 2001. Theoretical And Numerical Combustion. Edwards, Philadelphia, U.S.

[8] Veynant, D., Vervisch, L., 2002. "Turbulent combustion modelling". Progress in Energy and Combustion Science, 28 , pp. 193-266.

[9] Biagioli, F., Zimont, V.L., Syed, K.J., 2001. "Modelling and numerical simulation of gas combustion in dle burners based on the turbulent flame closure approach". Joint Power Generation conference, Paper JPGC2001/FACT19047.

[10] Bui-Pham, M. Seshadri, K. and Williams, F.A., 1992. "The asymptotic structure of premixed methane-air flames with slow co oxidation". Combustion and Flame, 89 , pp. 343362.

[11] F.C. Lockwood, N.G. Shah, 1981. "A new radiation solution method for incorporation in general combustion prediction procedures". In 18th International Symposium on Combustion, The Combustion Institute, Pittsburgh, pp. 1405-1416.

[12] W.M. Rohsenow, Y. Cho, J.P. Hartnett, 1998. Handbook of Heat Transfer, 2nd ed. McGraw-Hill Professional Publishing, New York, U.S.

[13] CD Adapco Group, 2001. Methodology STAR-CD VERSION 3.15. Computational Dynamics Limited.

[14] Lefebvre, A., 1983. Gas Turbine Combustion. Hemisphere Publishing Corporation, New York, U.S.

[15] F. Liu, H.A. Becker, Y. Bindar, 1998. "A comparative study of radiative heat transfer modelling in gas fired furnaces using the simple grey gas and the weigthed-sum-of-greygases models". Int. J. Heat Mass Transfer, 41 , pp. 33573371.

[16] H.C. Hottel, A.F. Sarofim, 1965. "The effect of gas flow patterns on radiative transfer in cylindrical furnaces". Int. J. Heat Mass Transfer, 8, pp. 1153-1169.

[17] N. Lallemant, A. Sayre and R. Weber, 1997. "Evaluation of emissivity correlations for $\mathrm{H}_{2} \mathrm{O}-\mathrm{CO}_{2}-\mathrm{N}_{2}$ /air mixtures and coupling with solution methods of the radiative transfer equation”. Prog. Energy Combust. Sci., 22 , pp. 543-574. 